\title{
Insulin resistance in women with hirsutism
}

Filiz Cebeci ${ }^{1}$, Nahide Onsun ${ }^{1}$, Meral Mert ${ }^{2}$

\begin{abstract}
1Department of Dermatology, Vakif Gureba Teaching Hospital, Istanbul, Turkey 2Department of Internal Medicine, Division of Endocrinology and Metabolism, Istanbul Medical Faculty, Istanbul University, Turkey
\end{abstract}

Submitted: 1 August 2010

Accepted: 20 October 2010

Arch Med Sci 2012; 8, 2: 342-346

DOI: 10.5114 /aoms.2012.28563

Copyright (c) 2012 Termedia \& Banach

\section{Abstract}

Introduction: There are still not enough data showing whether patients with idiopathic hirsutism $(\mathrm{IH})$ also have insulin resistance. The association between polycystic ovary syndrome (PCOS) and insulin resistance is well documented in the literature, but the Rotterdam Consensus has concluded that principally obese women with PCOS should be screened for the metabolic syndrome. We intended to investigate the presence/absence of insulin resistance in non-obese women with hirsutism.

Material and methods: Twenty-eight women with PCOS (14 non-obese and 14 obese), 12 non-obese with $\mathrm{IH}$, and 16 non-obese healthy women were included in the study. The presence of insulin resistance was investigated by using basal insulin levels and the homeostasis model assessment (HOMA) score in the study group.

Results: Patients with obese and nonobese PCOS had significantly $(p<0.05)$ higher basal insulin levels and HOMA scores than IH and control subjects. Insulin levels and HOMA scores did not differ between obese and non-obese PCOS patients. Patients with IH did not show any difference from the control group. Conclusions: Insulin resistance exists in non-obese women with PCOS as well as obese women with PCOS. The PCOS is associated with insulin resistance independent of obesity. Insulin resistance should be assessed in all hirsute women with PCOS regardless of their body mass index. More studies in larger numbers of patients should be performed to investigate the role of insulin resistance in women with $\mathrm{IH}$.

Key words: insulin resistance, idiopathic hirsutism, non-obese women.

\section{Introduction}

Hirsutism is characterized by excessive growth of terminal hair in the androgen-sensitive skin regions. It may result from various causes including polycystic ovary syndrome, non-classic adrenal hyperplasia, adrenal or ovarian tumors, or it may be idiopathic. Idiopathic hirsutism $(\mathrm{IH})$ is the second most common cause of hirsutism, after polycystic ovary syndrome [1]. Idiopathic hirsutism is defined as hirsutism associated with normal ovulatory function and normal circulating serum androgen concentrations [2]. In these patients mild forms of ovarian and adrenal functional hyperandrogenism may be present [3]. Polycystic ovary syndrome (PCOS) is characterized by chronic anovulation, obesity and hyperandrogenism. The Rotterdam Consensus Conference [4] proposed that the syndrome can be diagnosed after the exclusion of other disorders (non-classical adrenal hyperplasia, Cushing's syndrome, hyperprolactinaemia, hypothyroidism,

\author{
Corresponding author: \\ Meral Mert MD \\ Department \\ of Internal Medicine \\ Division of Endocrinology \\ and Metabolism \\ Istanbul Medical Faculty \\ Istanbul University \\ Turkey \\ Phone: 00905322915413 \\ E-mail: \\ meralmert@hotmail.com
}


virilizing adrenal or ovarian neoplasm), and the determination that at least two of the following are present: oligoovulation or anovulation, elevated levels of circulating androgens and/or clinical hyperandrogenism, and polycystic ovaries as defined by ultrasonography. Moreover, the Rotterdam Consensus has concluded that principally obese women with PCOS should be evaluated for the metabolic syndrome. It is well known that hyperandrogenism and insulin resistance are now closely associated. However, there are not enough data showing whether patients with $\mathrm{IH}$ also have insulin resistance.

Our aim was to investigate the presence/absence of insulin resistance and hyperinsulinaemia in especially non-obese women presenting with hirsutism.

\section{Material and methods}

Four groups of subjects were recruited: 14 obese (body mass index [BMI] $>27 \mathrm{~kg} / \mathrm{m}^{2}$ ) women with PCOS, 14 non-obese $\left(\mathrm{BMI}<25 \mathrm{~kg} / \mathrm{m}^{2}\right)$ women with PCOS, 12 non-obese women with $\mathrm{IH}$, and 16 nonobese controls. The study group comprised patients who attended the dermatology outpatient clinic. All patients were consulted by the Department of Endocrinology. The control subjects were matched for age and weight to the women with PCOS and $\mathrm{IH}$.

Obesity was defined as BMI $>27 \mathrm{~kg} / \mathrm{m}^{2}$. The diagnosis of PCOS was performed according to the Rotterdam 2003 criteria [4]. Rotterdam diagnostic criteria are shown in Table I. Patients with PCOS fulfilled all three Rotterdam criteria. The group with PCOS had oligomenorrhoea, polycystic ovarian morphology on ultrasonography and hyperandrogenemia estimated by laboratory measurements. Hyperandrogenemia was defined as a serum free testosterone level greater than $3.9 \mathrm{pg} / \mathrm{ml}$ (normal: 0.04-3.9 pg/ml). Oligomenorrhoea was defined as bleeding at intervals of greater than 35 days. Amenorrhea was defined as absence of menstruation for 6 months or more. Transabdominal or transvaginal ovary ultrasound was performed for all patients in the early follicular phase (days 3-9 of the menstrual cycle). Ovaries were classified as polycystic based on the presence of 12 or more follicles in each ovary measuring 2-8 $\mathrm{mm}$ in diameter, and/or increased ovarian volume (> $10 \mathrm{ml}$ ) [4]. Thyroid dysfunction, hyperprolactinaemia, non-classic congenital adre- nal hyperplasia, and adrenal/ovarian tumors were excluded by appropriate tests and ultrasonography of the ovaries and the adrenal glands. In the confirmed PCOS group, any patient with a $17 \alpha$-hydroxyprogesterone level of $>2 \mathrm{ng} / \mathrm{ml}$ (normal: 0.2$2.6 \mathrm{ng} / \mathrm{ml}$ ) was subjected to an adrenocorticotropic hormone stimulation test $(250 \mu \mathrm{g} I V$, Synachten; Ciba-Geigy, Basel, Switzerland) to rule out non-classic congenital adrenal hyperplasia. Hirsutism was defined as Ferriman-Gallwey (FG) score > 8 [5]. The diagnosis of $\mathrm{IH}$ was based on the presence of hirsutism, regular ovulatory menstrual cycles, and a normal serum androgen profile including free testosterone (normal: 0.04-3.9 pg/ml) and dehydroepiandrosterone sulfate (normal: $35-430 \mu \mathrm{g} / \mathrm{dl}$ ). All controls had a regular cycle and no clinical or laboratory features of PCOS. There was no history of diabetes mellitus in the study group. All participants had not used any medication or oral contraceptives for the last three months.

In the study group, blood sampling was done in the early follicular phase of spontaneous or induced (by medroxyprogesterone acetate $10 \mathrm{mg} /$ day for 7 days) menstrual cycles. After an overnight fast, blood samples were collected around 8-10 a.m. to measure serum levels of free testosterone (FT), dehydroepiandrosterone sulfate (DHEAS), prolactin (PRL), 17 $\alpha$-hydroxyprogesterone (17-OHP), fasting plasma glucose and insulin. Serum levels of DHEAS and PRL were measured by electrochemiluminescence immunoassays (Elecsys, Roche Diagnostic Corporation, Germany). Serum levels of FT and 17-OHP were measured by a radioimmunoassay kit (DPC, CA, USA). Plasma glucose levels were determined by the glucose oxidase technique with a glucose analyser (Dimension, Dade Behring, Germany). Plasma insulin concentrations were measured by an insulin radioimmunoassay kit (DPC, CA, USA); normal range $0-30 \mu \mathrm{lU} / \mathrm{ml}$; inter-assay $\mathrm{CV}<5 \%$; intra-assay CV $<8.5 \%$. Insulin resistance was calculated with homeostasis model assessment of insulin resistance (HOMA-IR), using the following formula: fasting serum insulin $(\mu \mathrm{U} / \mathrm{ml}) \times$ fasting plasma glucose $\mathrm{mmol} / \mathrm{l}) / 22.5$ [6].

\section{Statistical analysis}

Data analysis was performed using the SPSS 11 software (Chicago, IL, USA). The distribution of variables was tested for normality. The results are report-

Table I. 2003 Consensus Conference diagnostic criteria

\begin{tabular}{|l|}
\hline 1. Oligo- or anovulation \\
\hline 2. Clinical and/or biochemical hyperandrogenism findings \\
\hline 3. With polycystic ovarian appearance with exclusion of other causes \\
\hline The presence of at least two of three diagnostic criteria is essential \\
\hline
\end{tabular}


ed as means $\pm S D$, ranges (min-max), median values and interquartile range (IQR). The results of women with PCOS, obese and non-obese, were compared using the Mann-Whitney $U$ test. As continuous variables were without normal distribution, we used nonparametric tests. The comparisons of the non-obese groups were made by the Kruskal-Wallis test. Value of $p<0.05$ was regarded as statistically significant.

\section{Results}

Non-obese patients (PCOS, IH) and non-obese control subjects did not differ in mean age (22.6 $\pm 4.1,26.2 \pm 7.7$, and $28.0 \pm 6.5$ years, respectively $p>0.05$; range: $18-30$ for non-obese PCOS, $18-40$ for non-obese $\mathrm{IH}$ and 18-39 for control subjects) and BMI $\left(22.9 \pm 2.1,22.6 \pm 2.7\right.$, and $21.6 \pm 2.3 \mathrm{~kg} / \mathrm{m}^{2}$, respectively $p>0.05$ ) values. The Ferriman-Gallwey score was significantly higher in both women with PCOS and women with $\mathrm{IH}$ compared with controls $(16.7 \pm 10.2,11.4 \pm 2.7,4.3 \pm 1.2$, respectively $p<0.05)$.

Fasting glucose levels were similar in all groups and there were no statistically significant differences. However, patients with non-obese PCOS had significantly higher basal insulin levels and HOMA scores than patients with $\mathrm{IH}$ and control subjects $(p<0.05)$. Insulin levels and HOMA scores in patients with $\mathrm{IH}$ did not show any differences from the control subjects $(p>0.05)$. Age, BMI, fasting glucose, insulin, and HOMA values of the nonobese study group are shown in Table II. The women with non-obese PCOS had higher levels of insulin as well as women with obese PCOS and HOMA values were similar in both groups $(p>0.05)$. Age, BMI, fasting glucose, insulin, and HOMA values of groups of obese and non-obese with PCOS are shown in Table III.

\section{Discussion}

Polycystic ovary syndrome and $\mathrm{IH}$ account for most cases of hirsutism [1]. Not all hirsute patients have evidence of detectable androgen excess or endocrine imbalance, as in women with $\mathrm{IH}$. The association of carbohydrate metabolism abnormalities with androgen excess disorders, particularly PCOS, is a well-defined entity. Recognition of the relationship between PCOS and insulin excess dates to $1980[7,8]$. Insulin resistance and hyperinsulinism are now recognized as important features of PCOS, stimulating ovarian androgen secretion and suppressing sex hormone-binding globulin production [8, 9]. Insulin resistance and hyperinsulinaemia contribute to ovarian and adrenal cytochrome $\mathrm{P}^{450} \mathrm{C}_{\mathrm{C}} 17_{\alpha}$ activity in women with PCOS. Dysregulation of cytochrome $\mathrm{P} 450_{c} 17_{\alpha}$ enzyme causes the exaggerated secretion of adrenal and ovarian androgens in these women $[9,10]$.

Obesity is encountered in $30-70 \%$ of PCOSaffected women, and its presence significantly modifies both clinical and laboratory expression of the syndrome. Obesity increases the risk of co-morbidities associated with PCOS, such as impaired glucose tolerance and type 2 diabetes mellitus, hyperlipidaemia and arterial hypertension [11]. Weight loss is the first choice recommendation for the

Table II. General features of the non-obese study group

\begin{tabular}{|c|c|c|c|c|c|}
\hline & Age [years] & $\mathrm{BMI}\left[\mathrm{kg} / \mathrm{m}^{2}\right]$ & $\begin{array}{c}\text { Insulin }(0-30) \\
{[\mu \mathrm{U} / \mathrm{ml}]}\end{array}$ & $\begin{array}{c}\text { Glucose }(70-110) \\
{[\mathrm{g} / \mathrm{dl}]}\end{array}$ & HOMA \\
\hline \multicolumn{6}{|c|}{ Non-obese PCOS $(n=14)$} \\
\hline Mean \pm SD & $22.64 \pm 4.16$ & $22.96 \pm 2.14$ & $20.79 \pm 20.57$ & $88.57 \pm 7.86$ & $4.49 \pm 4.17$ \\
\hline Median (IQR) & $23(3.63)$ & 22.9 (1.78) & 12.05 (8.94) & $86.5(6.88)$ & $2.76(2.22)$ \\
\hline Min-max & $18-30$ & $20.0-26.66$ & $4.0-77.8$ & $75-99$ & $0.85-14.41$ \\
\hline \multicolumn{6}{|c|}{ Non-obese IH $(n=12)$} \\
\hline Mean \pm SD & $26.25 \pm 7.70$ & $22.64 \pm 2.73$ & $9.27 \pm 5.17$ & $85.17 \pm 9.13$ & $1.96 \pm 1.19$ \\
\hline Median (IQR) & $25.0(7.25)$ & $22.78(2.66)$ & $8.2(5.1)$ & $85.5(3.75)$ & 1.8 (1.01) \\
\hline Min-max & $18-40$ & $17.91-26.42$ & $3.0-18.4$ & $61-100$ & $0.61-4.54$ \\
\hline \multicolumn{6}{|c|}{ Non-obese control $(n=16)$} \\
\hline Mean \pm SD & $28.0 \pm 6.51$ & $21.60 \pm 2.38$ & $8.78 \pm 2.13$ & $81.63 \pm 10.12$ & $1.77 \pm 0.49$ \\
\hline Median (IQR) & $29.0(4.75)$ & $21.89(1.75)$ & $9.1(1.93)$ & $85.0(9.0)$ & $1.79(0.40)$ \\
\hline Min-max & $18-39$ & $17.26-24.97$ & $5.4-12.0$ & $62-94$ & $0.92-2.56$ \\
\hline \multicolumn{6}{|c|}{ Kruskal-Wallis test } \\
\hline$\chi^{2}$ & 5.074 & 2.035 & 6.034 & 2.700 & 7.853 \\
\hline$p$ & 0.079 & 0.361 & $0.049^{\star}$ & 0.259 & $0.020^{*}$ \\
\hline
\end{tabular}

${ }^{*} p<0.05$ 
Table III. General features of obese and non-obese groups with PCOS

\begin{tabular}{|c|c|c|c|c|c|}
\hline & Age [years] & $\mathrm{BMI}\left[\mathrm{kg} / \mathrm{m}^{2}\right]$ & $\begin{array}{l}\text { Insulin }(0-30) \\
{[\mu \mathrm{U} / \mathrm{ml}]}\end{array}$ & $\begin{array}{c}\text { Glucose (70-110) } \\
{[\mathrm{g} / \mathrm{dl}]}\end{array}$ & HOMA \\
\hline \multicolumn{6}{|c|}{ Obese PCOS $(n=14)$} \\
\hline Mean \pm SD & $24.36 \pm 5.99$ & $30.86 \pm 2.3$ & $20.72 \pm 1.55$ & $90.29 \pm 8.19$ & $4.71 \pm 3.99$ \\
\hline Median (IQR) & $23(4.63)$ & $30.11(1.75)$ & $17.2(5.5)$ & $90.5(5.63)$ & $3.68(1.44)$ \\
\hline Min-max & $18-40$ & $28.80-36.39$ & $7.3-69$ & $77-103$ & $1.62-17.55$ \\
\hline \multicolumn{6}{|c|}{ Non-obese PCOS $(n=14)$} \\
\hline Mean \pm SD & $22.64 \pm 4.16$ & $22.96 \pm 2.14$ & $20.79 \pm 20.57$ & $88.57 \pm 7.86$ & $4.49 \pm 4.17$ \\
\hline Median (IQR) & $23(3.63)$ & $22.9(1.78)$ & $12.05(8.94)$ & $86.5(6.88)$ & $2.76(2.22)$ \\
\hline Min-max & $18-30$ & $20-26.66$ & $4-7.8$ & $75-99$ & $0.85-14.41$ \\
\hline \multicolumn{6}{|c|}{ Mann-Whitney $U$ test } \\
\hline$Z$ & 0.487 & 4.521 & 0.851 & 0.621 & 0.965 \\
\hline$p$ & 0.626 & $0.0001^{*}$ & 0.395 & 0.534 & 0.335 \\
\hline
\end{tabular}

${ }^{*} p<0.001$

treatment of clinical manifestations of PCOS, such as menstrual cycle irregularities, infertility or hirsutism [11, 12].

Ehrmann et al. [13] showed that women with PCOS have a substantially higher impaired glucose tolerance and diabetes mellitus than age- and body weight-matched healthy women. The Rotterdam Consensus has concluded that principally obese women with PCOS should be evaluated for the metabolic syndrome. Obesity itself is usually accompanied by insulin resistance. Therefore, we intended to investigate the presence/absence of insulin resistance in women with especially non-obese PCOS and non-obese $\mathrm{IH}$. In the present study, significantly higher insulin levels and an insulin-resistant state were found in obese and non-obese women with PCOS. Studies on the relationship between obesity and hyperinsulinism in PCOS have yielded conflicting results. Chang et al. [14] and Dunaif et al. $[15,16]$ have demonstrated a degree of decreased insulin sensitivity in both obese and non-obese women by different methods. But others have not been able to find any degree of insulin resistance in non-obese women with PCOS [17-19]. In a recent study, it was demonstrated that nonobese women with PCOS have insulin resistance [20]. In our study, non-obese women with PCOS had significantly higher basal insulin levels and HOMA scores than non-obese women with $\mathrm{IH}$ and controls. However, there was no statistically significant difference with regard to insulin levels and HOMA scores between obese and non-obese women. On the other hand, we did not find any differences between obese women and non-obese women with respect to insulin levels and HOMA scores. Insulin levels were significantly higher in non-obese PCOS. Our findings showed that insulin resistance devel- ops independently from obesity in all hirsute women with PCOS. More recently, some studies have suggested that there may be a link between insulin resistance and hirsutism rather than body weight in women with PCOS [21, 22].

Insulin also seems to be one of the factors that interact with androgen to regulate pilosebaceous unit development [8]. The effect of insulin and the IGF system have been investigated by an in vitro study [23]. Some authors have shown that insulin/IGF plays a role in stimulating hair follicle growth acting together with androgens [24]. The pathophysiology of $\mathrm{IH}$ is presumed to involve a primary increase in skin $5 \alpha$-reductase activity and possibly an alteration in androgen receptor function [2]. In these patients there may be present mild forms of ovarian and adrenal functional hyperandrogenism [3]. In the light of this information, we investigated whether this peripheral increased androgen activity is associated with insulin metabolism. In contrast, there are not enough data showing whether patients with $\mathrm{IH}$ also have insulin resistance. Paoletti et al. [25] suggested that peripheral activity of androgens is related to hyperinsulinaemia and that anti-androgen treatment improves hyperinsulinaemia. Unlühizarci et al. [26] found a higher prevalence (18.7\%) of impaired glucose tolerance among women with $\mathrm{IH}$, and these authors also suggested that $\mathrm{IH}$ is associated with insulin resistance. Recently, insulin resistance was shown in obese patients with $\mathrm{IH}$ in another study [27]. In our study, the insulin levels and insulin-resistant state of women with non-obese $\mathrm{IH}$ and the non-obese control group did not show any differences. This might have been due to the small number of women with $\mathrm{IH}$.

In conclusion, insulin resistance should be assessed in all hirsute women with PCOS regard- 
less of their body mass index. More studies in larg er numbers of patients should be performed to investigate the role of insulin resistance in women with $\mathrm{IH}$.

\section{References}

1. Aziz R, Sanchez LA, Knochenhauer ES, et al. Androgen excess in women: experience with over 1000 consecutive patients. J Clin Endoc Metab 2004; 89: 453-62.

2. Aziz R, Carmina E, Sawaya ME. Idiopathic hirsutism. Endoc Rev 2000; 21: 347-62.

3. Rossi R, Tauchmanoca L, Luciano A, et al. Functional hyperandrogenism detected by corticotropin and $\mathrm{GnRH}$ analoque stimulation tests in women affected by apparently idiopathic hirsutism. J Endocrinol Invest 2001; 24: 491-8.

4. Rotterdam ESHRE/ASRM-sponsored PCOS Consensus Workshop Group. Revised consensus on diagnostic criteria and long term healty risks related to polycystic ovary syndrome. Human Reprod 2004; 19: 41-7.

5. Ferriman D, Gallwey JD. Clinical assessment of body hair growth in women. J Clin Endoc Metab 1961; 21: 1440-7.

6. Matthews DR, Hosker JP, Rudenski AS, et al. Homeostasis model assessment: insulin resistance and beta-cell function from fasting plasma glucose and insulin concentrations in man. Diabetologia 1985; 28: 412-9.

7. Burghen G, Givens J, Kitabchi A. Correlation of hyperandrogenism with hyperinsulinemia in polycystic ovarian disease. J Clin Endocrinol Metab 1980; 50: 113-6.

8. Rosenfield RL. Polycystic ovary syndrome and insulinresistant hyperinsulinemia. J Am Acad Dermatol 2001; 45: 95-104.

9. Dunaif A. Insulin action in the polycystic ovary syndrome. End Metab Clin Nort Am 1999; 28: 341-59.

10. Rosenfield RL, Barnes RB, Cara JF, et al. Dysregulation of cytochrome P450c17alpha as the cause of polycystic ovary syndrome. Fertil Steril 1990; 53: 785-91.

11. Vrbikova J, Hainer V. Obesity and polycystic ovary syndrome. Obes Facts 2009; 2: 26-35.

12. O'Connor A, Gibney J, Roche HM. Metabolic and hormonal aspects of polycystic ovary syndrome: the impact of diet. Proc Nutr Soc 2010; 10: 1-8.

13. Ehrmann DA, Barnes RB, Rosenfield RL, et al. Prevalence of impaired glucose tolerance and diabetes in women with polycystic ovary syndrome. Diabetes Care 1999; 22: 141-6.

14. Chang RJ, Nakamura RM, Judd HL, et al. Insulin resistance in nonobese patients with polycystic ovarian disease. J Clin Endocrinol Metab 1983; 57: 356-9.

15. Dunaif A, Segal KR, Futterweit W, et al. Profound peripheral insulin resistance, independent of obesity, in polycystic ovary syndrome. Diabetes 1989; 38: 1165-73.

16. Dunaif A, Segal KR, Shelley DR, et al. Evidence for distinctive and intrinsic defects in insulin action in polycystic ovary syndrome. Diabetes 1992; 41: 1257-66.

17. Dale PO, Tomb T, Voalir S, et al. Body weight, hyperinsulinemia, and gonadotrophin levels in the polycystic ovary syndrome: evidence of two distinct populations. Fertil Steril 1992; 58: 487-91.

18. Vrbikova J, Cibula D, Dvorakova K, et al. Insulin sensitivity in women with polycystic ovary syndrome. J Clin Endocrinol Metab 2004; 89: 2942-5.

19. Ketel IJ, Stehouwer CD, Serné EH, et al. Obese but not normal-weight women with polycystic ovary syndrome are characterized by metabolic and microvascular insulin resistance. J Clin Endocrinol Metab 2008; 93: 3365-72.
20. Takeuchi T, Tsutsumi O, Taketani Y. Abnormal response of insulin to glucose loading and assessment of insulin resistance in non-obese patients with polycystic ovary syndrome. Gynecol Endocrinol 2008; 24: 385-91.

21. Arthur LS, Selvakumar R, Seshadri MS, et al. Hyperinsulinemia in polycystic ovary disease. J Reprod Med 1999; 44: 783-7.

22. Amato MC, Galluzzo A, Merlino S, et al. Lower insulin sensitivity differentiates hirsute from non-hirsute Sicilian women with polycystic ovary syndrome. Eur J Endoc 2006; 155: 859-65.

23. Philpott MP, Sanders DA, Kealey T. Effects of insulin and insulin-like growth factors on cultured human hair follicles: IGF-I at physiologic concentrations is an important regulator of hair follicle growth in vitro. J Invest Dermatol 1994; 102: 857-61.

24. Itami S, Kurata S, Takayasu S. Androgen induction of follicular epithelial cell growth is mediated via insulin-like growth factor-I from dermal papilla cells. Biochem Biophys Res Commun 1995; 212: 988-94.

25. Paoletti AM, Cagnacci A, Orru M, et al. Treatment with flutamide improves hyperinsulinemia in women with idiopathic hirsutism. Fertil Steril 1999; 72: 448-53.

26. Unlühizarci K, Karababa $Y$, Bayram $F$, et al. The investigation of insulin resistance in patients with idiopathiv hirsutism. J Clin Endocrinol Metab 2004; 89: 2741-4.

27. Abdel Fattah NS, Darwish YW. Is there a role for insulin resistance in nonobese patients with idiopathic hirsutism? Br J Dermatol 2009; 160: 1011-5. 